else." It must be integrated in the staff development strategy and regular work life, e.g. by inserting mobility windows into the annual feedback meetings between executives and employees, or by including regular time slots for preparation to trainings and courses, as well as mobility activities in staff contracts. Internationalization activities for the administrative staff (e.g. language and intercultural courses, participation in mobility programs, staff weeks) must be closely integrated into a differentiated and systematic framework of staff development. HEIs should base their programs on information on the predispositions, prior knowledge, and experiences of their administrative staff. Activities such as mobility programs should explicitly target nonacademic staff as a particular group. We need to allow for, and support, bottom-up initiatives of staff related to skill development activities.

This needs more coherent HR structures, such as a systematic follow-up of internationalization activities for administrative staff in order to stimulate organizational learning in the HEI, and integrating different internationalization activities into structured programs. The SprInt program at Technische Universität Dresden is a good example, where a certificate consists of a language course, an intercultural course, and an optional mobility stay.

When it comes to internationalization today, nonacademic staff can be described as a crucial group, whose performance can significantly improve with the right measures of targeted recruitment and well-planned HR development activities.

\section{The Rise and Fall of Brazil's Science Without Borders}

\section{Creso M. SÁ}

Creso M. Sá is professor of Higher Education and director of the Centre for the Study of Canadian and International Higher Education, Ontario Institute for Studies of Education, University of Toronto, Canada. E-mail:c.sa@utoronto.ca.

B razil's Science Without Borders program attracted considerable attention when it was launched in 20II, with the promise to support study abroad opportunities for IOI,০०० students in STEM fields over four years. Spearheaded by none other than President Dilma Rousseff with an initial budget of US\$I.2 billion, the program seemed to represent a bold investment. Brazil has a longstanding shortage of STEM graduates and a largely insular higher ed- ucation system. With its explicit focus on placing students in highly ranked universities, some regarded the program as an important initiative to promote internationalization.

Less than five years on, the program's future hangs in the balance. Devaluation of the Brazilian real against the US dollar, along with constraints in the national budget, have led to the suspension of funding for the program in the fall of 20I5. Some awards granted were cancelled, and the program only continued to support students who were already abroad. There is a good chance Science Without Borders will not continue in its current form. However, the current budgetary situation is not the only problem afflicting the program, and hard questions need to be asked about what it has accomplished.

\section{The first problem this posed was of ac- tual demand: it soon became evident that English proficiency among the un- dergraduate population was generally low.}

\section{Questionable Policy Design}

Science Without Borders was created as a presidential initiative. There was no consultation process or public deliberation on the program priorities or design. Despite the longstanding role of federal agencies in managing scholarship programs for graduate study domestically and abroad, Science Without Borders came to life in a radically different size and format than preexisting initiatives. The most striking feature of the program is the focus on undergraduate students. Nearly 79 percent of all Science Without Borders awards were "sandwich" scholarships that support a year of study abroad for undergraduates. This brought an entirely new focus to the federal agencies in charge of graduate study and research.

The first problem this posed was of actual demand: it soon became evident that English proficiency among the undergraduate population was generally low. Federal agencies had to scramble to arrange language training for otherwise qualified students, which became rationalized as an initiative to "facilitate access" to study abroad opportunities (see http://isf.mec.gov.br). Evidently, no serious analysis of the candidate pool was undertaken prior to the implementation of the program, leading to the improvised effort to provide support for language learning.

Another demand issue came from the private sector. Industry was expected to fund 26,000 scholarships toward the total target, but that never materialized. Disagreements 
over goals and objectives between potential corporate sponsors and federal agencies led many companies to withdraw support.

Fundamentally, the undergraduate sandwich scholarship lacks any real articulation with students' home institutions and academic programs. Several problems arise from this: students have been admitted to colleges in fields other than their own, have taken unrelated coursework, and have not had their courses at foreign universities recognized for academic credit at their home institutions.

\section{The Student Experience}

Science Without Borders presented a formidable administrative challenge to the administering agencies. The agencies were not prepared to meet the sheer volume of applications and grantees brought about by Science Without Borders, which left them incapable to provide individual assistance to students and to properly monitor and manage individual files. There have also been recurring issues with the timely payment of student stipends, relocation grants, and tuition fees, creating serious difficulties for some grantees.

\section{Science Without Borders presented a formidable administrative challenge to the administering agencies.}

A study by Julieta Grieco at the University of Toronto, the institution that has the largest number of Science Without Borders grantees, examined the experience of undergraduate students in detail. In general, students lacked proper predeparture orientation in Brazil to better prepare them to the new academic and culture contexts. They also lacked effective academic advising abroad, to help them access opportunities at the host institution and to navigate the academic system.

Differences in the structure of academic programs lead students to take classes at an inappropriate level, either for lack of knowledge of how the curriculum works or because they lacked course requirements for higher-level courses. A major stumbling block for some students is the inability to gain admission to schools and departments offering their program at the host university. This is often the case with students in professional undergraduate programs in Brazil that are only taught as graduate programs in North America (e.g. medicine). Such students are channeled to the general liberal arts curriculum, where they take classes that are unrelated to the coursework of their home programs.

Science Without Borders allows students to engage in industry or research internships. However, there is no general coordination of this aspect of the program and no clear guidance to students, who receive uneven assistance with how to access companies or academic laboratories that might provide placement opportunities. While some students have positive experiences with coursework and internships, it is evident that success is to a great extent left to chance.

\section{Lack of Policy Learning}

The lack of a national culture of policy evaluation is evident in the case of Science Without Borders. The risks of policy failure, wasteful spending, and adverse unintended consequences are clear with a program of this magnitude. Unfortunately, no mechanism is in place to monitor and assess this program, and generate useful policy lessons.

Science Without Borders provides a stark example of lacking policy capacity in the Brazilian government to design and implement effective public policy. Opaque decision-making about key program features, absence of consultation with key stakeholders, and top-down implementation have resulted in a poorly designed program. The major flaws in design discussed above were all avoidable. Nonetheless, no effort was made to assess existing needs and demands among students, administering agencies, universities, and potential industry partners.

\section{Establishing Real Priorities}

Science Without Borders consumed significant resources that could have been better employed elsewhere. Debate about the relative merit of allocating 6.4 Brazilian reals to this program never happened. This was a consequential decision for the academic research system. Funding for Science Without Borders was not "new money," but rather a reallocation of resources supporting university researchers and graduate students. This resulted in budget cuts and delays in spending that affected research programs around the country. These implications need to be considered as part of the opportunity costs of this program.

A revival of Science Without Borders in its original format is unlikely. The program's undergraduate focus is hard to justify academically, and finds political opponents among opposition parties. Given the president's association with the program, the government is not likely to simply terminate it, which would signal failure. A silent downsizing and shift in focus to graduate training through the budgetary process is more probable. 